\title{
Evidência de Validade do Inventário da Consciência Metacognitiva (ICM Jr) para Uso no Brasil
}

\author{
Validity Evidence of the Junior Metacognitive Awareness Inventory (ICM Jr) for Use \\ in Brazil
}

\author{
Geisiele de Souza Teotonio ${ }^{1}$, Geida Maria Cavalcanti de Sousa ${ }^{2}$, Leonardo Rodrigues Sampaio ${ }^{3}$, \\ Nilton Soares Formiga ${ }^{4}$ e Geazi Rosa Oliveira Teotonio 5
}

\begin{abstract}
Resumo
O objetivo desse estudo foi traduzir, adaptar e validar o Inventário da Consciência Metacognitiva (ICM-Jr), criado por Sperling, Howard, Miller e Murphy (2002), para uso no Brasil. 698 estudantes dos ensinos fundamental e médio das cidades de Juazeiro-BA e Petrolina-PE compuseram as amostras de dois estudos. No primeiro observaram-se evidências de validade fatorial e consistência interna da versão traduzida e adaptada do instrumento, sugerindo sua adequação para avaliação da metacognição em adolescentes brasileiros. As análises a respeito da dimensionalidade da escala corroboram o modelo bifatorial de Sperling et al. (2002), composto pelos fatores "Conhecimento da cognição" e "Regulação da cognição". Por fim, estudantes do ensino médio obtiveram escores mais elevados que os do ensino fundamental, quando se considerou apenas o período intermediário da adolescência. Os resultados são discutidos a partir de estudos recentes sobre metacognição, além de considerações a respeito da realidade sócioeducacional do contexto da pesquisa.
\end{abstract}

Palavras-chave: metacognição, adaptação, validade fatorial, adolescentes

\begin{abstract}
The aim of the current research was to translate, to adapt and to validate the Metacognitive Consciousness Inventory (ICM-Jr), by Sperling, Howard, Miller, and Murphy (2002), for its use in Brazil. 698 students enrolled in middle and high school levels, in the cities of Juazeiro-BA and Petrolina-PE, composed the samples of two studies. In the first study, validity evidences of the translated and adapted version of that instrument were found, suggesting its adequacy for evaluation of metacognition in Brazilian adolescents. Analyzes regarding the dimensionality of the scale corroborated the two-factor model of Sperling et al. (2002), composed by the factors "Knowledge of cognition" and "Regulation of cognition". Finally, 15- to 16year-old in High School scored higher than those in the Middle School. The results are discussed in the light of recent studies on metacognition and considering the socio-educational reality where the research took place.
\end{abstract}

Keywords: metacognition, adaptation, factorial validity, adolescents

\footnotetext{
${ }^{1}$ Mestre em Psicologia. Professora na Escola Manoel Nunes Barbosa. Povoado Poço da Cruz, s/n. Brasil. Tel.: +55(87)3862-3202. Email: ggbiologialove@ hotmail.com

${ }^{2}$ Doutora em Psicologia. Professora da Universidade Federal do Vale do São Francisco - UNIVASF. Av. José de Sá Maniçoba, s/n. 56304-970 Centro Petrolina - PE. Brasil. Tel.: +55(87)2101-6868. E-mail: geida.cavalcanti@univasf.edu.br

${ }^{3}$ Doutor em Psicologia Cognitiva. Professor da Universidade Federal do Vale do São Francisco - UNIVASF. Av. José de Sá Maniçoba, s/n. 56304-970 Centro Petrolina - PE. Brasil. Tel.: +55(87)2101-6868. E-mail: leonardo.sampaio@univasf.edu.br

${ }^{4}$ Doutor em Psicologia Social. Professor na Universidade Potiguar. Rua Francisco Brandão, 973. Bairro de Manaíra. 58038-520. João Pessoa-PB, Brasil. Tel: +55(83)986402562. E-mail: nsformiga@yahoo.com

${ }^{5}$ Mestre em Ciências. Técnico administrativo em Educação da Universidade Federal do Vale do São Francisco - UNIVASF Rodovia BR 407, Km 12, Lote 543. Brasil. Projeto de Irrigação Senador Nilo Coelho, s/nº-C1. Tel.: +55(87)2101-4872. E-mail: geazi.roteotonio@univasf.edu.br 


\section{Introdução}

A metacognição pode ser entendida como um mecanismo autorregulatório que permite aos indivíduos acessar pensamentos e conhecimentos relativos à foma como sua própria cognição atua (Flavell, 1979; Cannon-Bowers, Rhodenizer, Salas, \& Bowers, 1998). Assim, quando analisamos uma tarefa que está sendo realizada, se essa atingirá os objetivos propostos, estamos utilizando habilidades metacognitivas (Ribeiro, 2003; Figueira, 2003)

As habilidades metacognitivas são utilizadas em diversas situações do nosso cotidiano, como solucionar problemas, se comunicar, compreender oralmente uma informação, leitura, escrita, na aquisição da linguagem, na atenção, na memória, entre outros. Por esta razão, o conceito de metacognição assume importância para áreas como a Educação e a Psicologia (Flavell, 1979, Dunlosky \& Metcalfe, 2009; Ribeiro et al., 2015).

No que diz respeito especificamente à aprendizagem escolar, a metacognição ocorre no momento em que os alunos planejam, monitoram e avaliam seu próprio pensamento em um ambiente de aprendizagem ou de resolução de problemas (Ayersman, 1995). Esse monitoramento metacognitivo favorece a participação ativa do estudante no processo de aprendizagem, contribui para que ele consiga identificar suas deficiências e virtudes (em termos de capacidade para elaboração do conhecimento) e promove motivação para novas aprendizagens (Cannon-Bowers et al., 1998). Por esta razão, observa-se que alunos que apresentam bom desempenho acadêmico são mais hábeis na manipulação de estratégias metacognitivas do que alunos que apresentam baixo desempenho acadêmico (Boruchovitch, 1999). Além disso, alunos eficientes em executar atividades acadêmicas possuem também competências metacognitivas mais desenvolvidas, pois demonstram compreender o objetivo da atividade, planificar a sua realização, aplicar e alterar conscientemente estratégias de estudo e avaliar o seu próprio processo de execução (Flavell \& Wellman, 1977).

Segundo a perspectiva de Schraw e Dennison (1994), Davidson, Deuser e Sternberg (1994) e Schraw (1998), existem dois componentes principais da metacognição: conhecimento metacognitivo e habilidade metacognitiva, os quais também são referidos como o conhecimento da cognição e regulação da cognição, respectivamente. $\mathrm{O}$ conhecimento da cognição refere-se ao que os indivíduos sabem sobre a sua própria cognição, em três níveis diferentes: declarativo (saber sobre as coisas), procedimental (saber sobre como fazer as coisas) e condicional (saber por que e quando fazer as coisas). Já a regulação da cognição, refere-se às atividades e ações realizadas pelas pessoas para controlar a sua própria cognição, as quais são agrupadas em cinco categorias: planejamento, acompanhamento, gestão de informação, apuramento e avaliação. Essas competências reguladoras ajudam os estudantes a controlar a sua aprendizagem, orientam o processo de resolução de problemas e seu refinamento melhora a eficiência no processo de tomada de decisão.

Importante destacar que a metacognição segue um curso de desenvolvimento que se inicia ainda na infância e continua até a vida adulta, o que faz com que esta capacidade se torne cada vez mais explícita, poderosa e efetiva à medida que avança a idade e que o uso de estratégias metacognitivas é estimulado (Kuhn, 2000). A este respeito, Salles, Ais, Semelman, Sigman e Calero (2016) observaram que crianças (seis a nove anos) e adultos possuíam habilidades metacognitivas similares para resolver uma tarefa perceptiva, mas com a diferença de que elas demonstravam maior confiança na autoavaliação que faziam sobre seu próprio desempenho nas atividades. Ou seja, apesar de as crianças nesta idade já demonstrarem um acesso cognitivo similar ao de adultos, ainda falta-lhes desenvolver completamente a capacidade de monitorar acuradamente o resultado de suas próprias decisões, quando estão resolvendo tarefas que demandem o uso da metacognição.

Existem diversos instrumentos que avaliam o desenvolvimento metacognitivo, tais como entrevistas (Swanson, 1990; Zimmerman \& Pons, 1986), a monitorização através de listas de verificação (Manning, Glasner, \& Smith, 1996), além das escalas psicométricas. Boruchovitch et al. (2006), em uma pesquisa de revisão sobre instrumentos que se propõem a avaliar a metacognição, constaram que inicialmente a 
metacognição foi medida por escalas, inventários e entrevistas estruturadas, relativas às estratégias de aprendizagem de modo geral. Após algum tempo, o construto passou a ser avaliado por instrumentos que abrangiam outros contextos, como saúde mental e processos de memorização.

Dentre os instrumentos usados para avaliar a metacognição em adultos, encontra-se o Inventário de Consciência Metacognitiva (MAI Metacognitive Awareness Inventory), formulado por Shraw e Denisson (1994). O MAI avalia o conhecimento e a regulação cognitiva por meio de 52 itens, distribuídos entre dois fatores caracterizados como conhecimento e regulação cognitiva. Esse instrumento foi traduzido e adaptado para uso em países como Portugal (Ribeiro, Simões, \& Almeida, 2016), Turquia (Akin, Abaci, \& Çetin, 2007) e Colômbia (Bustos, Bravo, \& Leon, 2014), o que aponta para validade e confiabilidade do instrumento para mensuração da metacognição em diferentes contextos culturais.

Baseando-se no MAI, Sperling, Howard, Miller e Murphy (2002) desenvolveram o inventário da consciência metacognitiva Jr. (ICM Jr) versões A e B, objetivando, dentre outras coisas, propor um instrumento adequado para avaliar a eficiência de intervenções educacionais para o desenvolvimento da Metacognição. A versão A é adequada para mensuração do desenvolvimento metacognitivo em alunos do terceiro ao quinto ano de escolaridade, possuindo 12 itens que são avaliados por meio de escalas tipo likert de 3 pontos. Já a versão $B$ foi desenvolvida para avaliação de alunos mais experientes, com níveis mais altos de autorregulação, que estão entre o sexto a nono ano de escolaridade e possui 18 itens que são respondidos através de escalas de 5 pontos. Para a construção desse instrumento, Sperling e seus colaboradores consideraram os três componentes do conhecimento da cognição (declarativo, condicional e procedimental) e os três componentes da regulação da cognição (planificação, monitorização e avaliação), já avaliados no MAI.

O ICM Jr. tem sido utilizado amplamente em diversas investigações para avaliar o desenvolvimento metacognitivo, sendo um instrumento reconhecido principalmente para demonstrar a relação entre aquele construto e a aprendizagem (Narang \& Saini, 2013). Gonçalves, Fidalgo e Martins (2011) realizaram a tradução, adaptação e validação da versão $B$ deste instrumento em Portugal, com estudantes do sétimo, oitavo e novo anos de escolaridade, obtendo um instrumento com boa consistência interna geral (Alfa $=.85)$ e estrutura fatorial similar àquela observada por Sperling et al. (2002), que avalia o reconhecimento e a regulação da cognição de forma global por meio de 15 itens.

No que diz respeito especificamente ao Brasil, Pascualon (2011) e Lima Filho e Bruni (2015) realizaram a aplicação de questionários em diferentes contextos, com alunos do ensino fundamental II e com alunos graduandos e profissionais já graduados. Para tanto, esses autores adaptaram e validaram instrumentos de avaliação da metacognição. Joly, Santos e Marini (2006), por meio da Escala Metacognitiva de Leitura, e Marini e Joly (2008), aplicando a Escala de Estratégias de Leitura, avaliaram o tipo e a frequência de estratégias metacognitivas utilizadas na leitura no ensino médio e fundamental I, respectivamente. Oliveira, Boruchovitch e Santos (2009), por meio da Escala de Avaliação das Estratégias de Aprendizagem, avaliaram estratégias cognitivas e metacognitivas de aprendizagem de crianças do ensino fundamental, mas ressaltam que há carência de estudos que permitam evidenciar as propriedades psicométricas de instrumentos destinados a avaliar o desenvolvimento metacognitivo.

Apesar da importância dos estudos supracitados, observa-se que ainda há uma lacuna no que diz respeito a instrumentos adequados para mensuração da metacognição entre adolescentes brasileiros. Frente a isto, o presente estudo teve como objetivo traduzir, adaptar e validar a versão B do Inventário da Consciência Metacognitiva (ICM-Jr) à população brasileira e verificar o perfil metacognitivo de alunos do ensino fundamental (séries finais) e do ensino médio, ao longo de dois estudos. O primeiro buscou explorar a estrutura fatorial para reunir evidências de validade fatorial e consistência interna do ICM Jr., enquanto que o segundo buscou avaliar a dimensionalidade do construto Metacognição, por meio da Modelagem de Equações Estruturais, comparando o modelo bidimensional proposto por Sperling et al. (2002) 
com outros alternativos, afim de identificar aquele com os melhores indicadores psicométricos.

\section{Primeiro Estudo}

\section{Método}

\section{Amostra}

A amostra foi calculada através do pacote estatístico G Power 3.1, um software destinado a calcular o poder estatístico (isto é, o teste de hipótese), tendo como base, não apenas o ' $n$ ' necessário para a pesquisa, mas, também, o tipo de calculo a ser realizado (Faul, Erdfelder, Buchner, \& Lang, 2007). Para a coleta de dados deste primeiro estudo, foi considerada uma probabilidade de $95 \% \quad(p<.05)$, magnitude do efeito amostral ( $r \geq 30)$ e um padrão de poder hipotético $(\pi \geq .80)$.

A partir desses critérios, a amostra total foi composta por 434 adolescentes, com idades variando entre 12 e 18 anos $\left(\mathrm{M}_{\text {idade }}=14,77\right.$; $\mathrm{dp}=1.71)$, do sexo masculino $(\mathrm{n}=220-45 \%)$ e feminino $(\mathrm{n}=267-55 \%)$, matriculados do sexto à nona série do Segundo Ciclo do Ensino Fundamental $(n=260-53 \%)$ e nas três séries do Ensino Médio ( $\mathrm{n}=227-47 \%$ ), de escolas públicas $(\mathrm{n}=362-74 \%)$ e particulares $(\mathrm{n}=125-26 \%)$ das cidades brasileiras de Petrolina - PE $(n=227-$ $47 \%)$ e Juazeiro - BA $(n=260-53 \%)$. A referida amostra revelou-se suficiente para o estudo em questão, tendo como indicadores: $t \geq 1.98 ; \pi \geq .99$; $p<.05$.

\section{Instrumentos}

$\mathrm{O}$ instrumento utilizado nesse trabalho foi o Inventário da Consciência Metacognitiva Jr. (ICM Jr), versão B, criado por Sperling et al. (2002). Este instrumento é constituído por 18 itens que são avaliados por meio de escalas tipo Likert de 5 pontos (1=Nunca; 2=Raramente; 3=Às vezes; 4=Regularmente; $5=$ Sempre) e que mensuram os seguintes componentes da Metacognição: conhecimento da cognição e regulação da cognição.

O instrumento foi traduzido a partir da versão B do do ICM Jr. (Sperling et al., 2002) validado em Portugal (Gonçalves et al., 2011), com a participação de diferentes juízes: um profissional com doutorado e formação em Letras/Português, um profissional especializado da área de licenciatura, um pesquisador na área de Psicologia Cognitiva e um tradutor das línguas inglesa e portuguesa.

\section{Procedimentos}

Antes da aplicação foi realizado um pré-teste, buscando-se avaliar a adequação dos termos empregados no instrumento após sua tradução para o Português do Brasil. Para realização desse pré-teste foram selecionados 20 estudantes de escola privada da cidade de Petrolina-PE. Inicialmente foram dadas instruções quanto ao preenchimento dos dados, mas as questões não foram lidas em voz alta, nem foram explicados os sentidos globais das afirmações, sendo apenas esclarecidas dúvidas quanto ao significado de palavras isoladas. O pré-teste foi dividido em duas etapas: na primeira, os alunos responderam ao ICM Jr. individualmente e em seguida participaram de uma sessão do tipo grupo focal, quando expressaram suas opiniões sobre a escala. Após essas considerações, alguns termos e expressões do português de Portugal foram ajustados para a aplicação no Brasil (ex: "Eu tento usar formas de estudo que resultaram comigo anteriormente"; "Eu questiono-me sobre os meus progressos enquanto estou a aprender alguma coisa nova"; "Depois de terminar uma tarefa, interrogo-me se havia uma forma mais fácil de a fazer").

A aplicação da versão corrigida do ICM-Jr foi realizada no tempo médio de 10 minutos, no ambiente escolar, por pesquisadores previamente treinados para o processo de coleta de dados, que davam as instruções oralmente e garantiam total disponibilidade para esclarecer dúvidas dos participantes.

\section{Análises estatísticas}

Além de estatísticas descritivas, realizou-se uma Análise de Componentes Principais (ACP), não estabelecendo rotação, pois, com base nos estudos anteriores hipotetizava-se sua bifatorialização. Considerou-se previamente a possibilidade de realizar a ACP, tomando o KMO igual ou superior a .70 e o Teste de Esfericidade de Bartlett, com nível de significância de até 5\% (Tabachnick \& Fidell, 2012). 
Considerando que os critérios de Kaiser (valor próprio igual ou superior a 1) e Cattell (distribuição gráfica dos valores próprios) tendem a maximizar o número de fatores a extrair, decidiu-se, adicionalmente, efetuar uma análise paralela (Hayton, Allen, \& Scarpello, 2004; Ledesma \& Valero-Mora, 2007), trabalhando-se com a sintaxe do SPSS desenvolvida por O’Connor (2000). Por fim, foi calculada a consistência interna dos fatores resultantes, por meio do Alfa de Cronbach.

\section{Aspectos éticos}

Os dois estudos descritos neste trabalho faziam parte de um projeto global o qual foi aprovado pelo Comitê de Ética em Pesquisas com Seres Humanos da Univasf, antes de sua realização (Parecer $n^{\circ}$ 1648081). As escolas que participaram do estudo assinaram a Carta de Anuência, enquanto que os estudantes e seus responsáveis assinaram o Termo de Assentimento e o Termo de Consentimento Livre e Esclarecido (TCLE), respectivamente, garantindo o sigilo e confidencialidade dos participantes e dos dados coletados.

\section{Resultados}

Decidiu-se verificar, inicialmente, o poder discriminativo dos itens, o qual teve como objetivo apresentar uma maior especificidade na análise estatística para a organização e verificação empírica da estrutura fatorial do ICM Jr. Para tanto, buscou-se avaliar, a partir dos pressupostos da Teoria Clássica dos Testes (TCT), se os itens apresentavam capacidade de discriminar pessoas com magnitudes próximas, ou seja, discriminar aquelas dos grupos inferiores e superiores, em relação ao construto medido (Pasquali, 2003). Outro motivo para realizar este cálculo, deveu-se ao fato de não ter sido observado este tipo de análise nos estudos de Sperling et al. (2002), condição que contribui muito para antecipar a seleção dos itens para fatorialidade da medida em questão.

Neste sentido, foi calculada uma pontuação total da escala e a sua mediana, cujo valor foi 67 . Em seguida, os respondentes com pontuação abaixo da mediana foram classificados como sendo do grupo inferior, enquanto que aqueles com pontuações acima da mediana foram definidos como pertencentes ao grupo superior. Considerando-se cada um dos itens desta medida, efetuou-se um teste t para amostras independentes e compararam-se ambos os grupos, observando quais dos itens da escala discriminam as pessoas com magnitudes próximas. Os resultados deste teste demonstraram que todos os 18 itens apresentaram poder discriminativo em relação ao construto Metacognição (Quadro 1).

Quadro 1. Poder discriminativo dos itens da Escala de Metacognição

\begin{tabular}{|c|c|c|}
\hline Itens & $t$ & $\begin{array}{c}\text { Correlação item- } \\
\text { escala }\end{array}$ \\
\hline Item 1 & $-6.20 * *$ & $.37 * *$ \\
\hline Item 2 & $-7.22 * *$ & $.42 * *$ \\
\hline Item 3 & $-8.03 * *$ & $.45^{* *}$ \\
\hline Item 4 & $-5.22 * *$ & $.28 * *$ \\
\hline Item 5 & $-4.50 * *$ & $.33 * *$ \\
\hline Item 6 & $-8.65^{* *}$ & $.48^{* *}$ \\
\hline Item 7 & $-12.10 * *$ & $.59^{* *}$ \\
\hline Item 8 & $-10.62 * *$ & $.51 * *$ \\
\hline Item 9 & $-10.35^{* *}$ & $.53 * *$ \\
\hline Item 10 & $-8.02 * *$ & $.41^{* *}$ \\
\hline Item 11 & $-8.19 * *$ & $.40 * *$ \\
\hline Item 12 & $-4.53 * *$ & $.23 * *$ \\
\hline Item 13 & $-10.11 * *$ & $.54 * *$ \\
\hline Item 14 & $-11.35^{* *}$ & $.52 * *$ \\
\hline Item 15 & $-8.71 * *$ & $.41^{* *}$ \\
\hline Item16 & $-7.00 * *$ & $.26 * *$ \\
\hline Item 17 & $-7.57 * *$ & $.40^{* *}$ \\
\hline Item 18 & $-10.94 * *$ & $.56 * *$ \\
\hline
\end{tabular}

Além disso, optou-se por avaliar a relação do conteúdo dos itens com o construto Metacognição, tendo como objetivo verificar a representatividade comportamento-domínio, a qual, sistematicamente, avalia a relação teórica 
apresentada no instrumento de medida desenvolvido por Sperling et al. (2002) e as situações especificadas nos itens e o quanto este instrumento representa os aspectos esperados (Pasquali, 2003). A partir dessa perspectiva, aplicou-se o teste de Pearson (r) para avaliar a correlação dos itens do ICM-Jr. com o total da escala. Conforme pode ser observado no Quadro 1 , todos os 18 itens se correlacionaram significativamente com o total da escala. Todavia, os itens 4, 12 e 16 apresentaram correlação inferior a .30 , o que fez com que os mesmos fossem excluídos das análises subsequentes.

Com intuito de aprofundar as análises e produzir mais subsídios que contribuíssem com a tomada de decisão a respeito da quantidade de fatores a serem retidos, optou-se por realizar uma análise paralela, assumindo os mesmos parâmetros do banco de dados original, isto é, 434 participantes e 18 variáveis, tendo seus valores próprios gerados em 1.000 simulações aleatórias com os itens do ICM-Jr. Essa análise demonstra que nos quatro primeiros fatores os Eigenvalues obtidos a partir dos dados reais foram maiores do que 1. Porém, apenas em relação aos dois primeiros, além de superior a 1 os Eingevalues também foram maiores do que aqueles produzidos a partir das simulações aleatórias, razão esta que sugeriu a retenção apenas destas duas dimensões (O'Connor, 2000). Os valores dos Eigenvalues observados foram os seguintes: Fator 1 (Autovalor real $=3.51$; Autovalor aleatório $=1.41$ ), Fator 2 (Autovalor real =1.56; Autovalor aleatório $=1.32$ ), Fator 3 (Autovalor real =1.23; Autovalor aleatório $=1.29$ ) e Fator 4 (Autovalor real =1.05; Autovalor aleatório $=1.21$ ).

A partir destes resultados, partiu-se para uma análise de Componentes Principais (APC), sem fixar o número de fatores a serem extraídos e utilizando-se rotação do tipo Varimax, assumindose como critério uma saturação de .30 . Visando a segurança na tomada de decisão na escolha dos fatores, três critérios foram levados em conta: (1) quantidade de valores próprios (Eigenvalues) iguais ou superiores a 1 (Critério de Kaiser), (2) distribuição gráfica dos valores próprios, tomando como referência o ponto a partir do qual nenhum outro fator aporta consideravelmente para a estrutura (Critério de Cattell) e (3) análise paralela (Fabrigar, Wegener, MacCallum, \& Strahan,
1999; O’Connor, 2000; Hayton, Allen, \& Scarpello, 2004; Ledesma \& Valero-Mora, 2007).

Os resultados dessas análises permitiram identificar a adequação da matriz de correlação: $\mathrm{KMO}=.82$ e Teste de Esfericidade de Bartlett $\left(\chi^{2}=843.98\right.$ e g.l.=105, $\left.p<.001\right)$. A partir da distribuição gráfica (critério de Cattell) e da aplicação do critério de Kaiser, foi possível identificar a existência de quatro fatores na escala ICM-Jr, com Eigenvalues maiores que 1, os quais conjuntamente explicam cerca de $45 \%$ da variância total observada.

Todavia, considerando-se a dificuldade de interpretação teórica desses quatro fatores, os resultados da Análise Paralela, bem como os dados obtidos por Sperling et al. (2002) e por Gonçalves et al. (2011), optou-se por realizar uma nova ACP, fixando-se a solução final em dois fatores. Os resultados desta análise $(\mathrm{KMO}=.84 \mathrm{e}$ Teste de Esfericidade de Bartlett, com $\chi^{2}=843.98$ e g.l. $=105 ; p<.001)$ indicaram que os quinze itens restantes do ICM-Jr. se distribuíram nestes dois fatores, os quais foram responsáveis por explicar cerca de $31 \%$ da variância total observada.

$\mathrm{O}$ primeiro fator agrupou apenas itens relacionados à Regulação da Cognição, com exceção do item 14 ("Eu utilizo estratégias de aprendizagem diferentes de acordo com a tarefa"), originalmente pertencente à dimensão Conhecimento da Cognição. Por outro lado, o segundo fator agrupou seis itens relacionados ao componente Conhecimento da Cognição, e apenas um item da dimensão Regulação da Cognição (o item 11: "Eu presto realmente atenção às informações importantes").

Considerando essa distribuição de itens, optou-se por manter a denominação originalmente proposta por Sperling et al. (2002), definindo-se o primeiro fator como sendo o de Regulação da Cognição (RC) e o segundo de Conhecimento da Cognição (CC). Apesar das exceções observadas em relação à inclusão de itens não esperados nestes fatores, compreende-se que, de forma geral, os itens da escala avaliam uma dimensão mais ampla da metacognição e que as especificidades relacionadas às duas dimensões nomeadas por Sperling et al. (2002) podem não ter sido tão claramente definidas, quando da elaboração dos itens do ICM-Jr.

A escala final, após a eliminação destes três 
Quadro 2. Cargas fatoriais dos itens e indicadores psicométricos das dimensões do ICM-Jr

\begin{tabular}{lcc}
\hline Itens da Escala & RC* & CC** \\
\hline 6. Eu desenho esquemas ou faço resumos para me ajudar a compreender o assunto & .50 \\
7. Quando termino uma tarefa escolar, pergunto-me se aprendi o que queria & .64 \\
8. Eu penso em diversas formas de resolver um problema e depois escolho a melhor & .53 \\
9. Eu penso sobre o que preciso aprender antes de começar a estudar. & .52 \\
10. Eu me questiono sobre os meus avanços quando estou aprendendo alguma coisa nova & .58 \\
14. Eu utilizo planos/ estratégias de aprendizagem diferentes de acordo com a tarefa. & .54 \\
15. Eu costumo verificar como ocorre o meu estudo para ter certeza de que terminarei a tempo. & .52 \\
18. Eu decido o que preciso fazer antes de iniciar uma tarefa. & .51 \\
17. Depois de terminar uma tarefa, pergunto-me se havia uma forma mais fácil de fazê-la & .48 \\
1. Eu sei quando entendo alguma coisa & \multicolumn{2}{c}{.44} \\
2. Eu consigo aprender quando preciso. & \multicolumn{2}{c}{.58} \\
3. Eu tento usar formas de estudo que deram certo comigo antes & \multicolumn{2}{c}{.50} \\
5. Eu aprendo melhor quando já sei alguma coisa sobre o assunto & \multicolumn{2}{c}{58} \\
11. Eu presto realmente atenção às informações importantes. & .64 \\
13. Eu utilizo os meus pontos fortes para ultrapassar as minhas fraquezas. & \multicolumn{2}{c}{.62} \\
N $^{\circ}$ Itens & 8 & 6 \\
Valor próprio & 3.31 & 1.35 \\
Variância explicada (\%) & 22.1 & 9.08 \\
Média & 30.81 & 24.12 \\
Desvio-padrão & 6.18 & 3.57 \\
Alfa de Cronbach & .70 & .60 \\
* Regulação da Cognição. ** Conhecimento da Cognição & &
\end{tabular}

itens apresentou consistência interna aceitável (Alfa $=.74$ ), assim como as suas duas subdimensões (Quadro 2). Conforme defende Kline (2016), o valor de Alfa mais comumente aceitável para testes cognitivos é acima de .80, podendo-se esperar valores abaixo de .70 , quando se trata de construtos psicológicos.

Objetivando-se testar se o modelo bifatorial encontrado na análise exploratória do primeiro estudo é apropriado para explicar o construto da metacognição, decidiu-se por executar um segundo estudo com uso da Análise Fatorial Confirmatória e método de Modelagem de Equações Estruturais. Os resultados deste estudo serão apresentados a seguir.

\section{Segundo Estudo}

\section{Método}

\section{Amostra}

Participaram desse segundo estudo 211 estudantes, com idades entre 11 e 18 anos (Média=14.28, dp=1.93), do sexo feminino
(55.5\%), sendo $68.7 \%$ do ensino público, $61 \%$ do nível fundamental e 39\% do nível médio, oriundos de escolas públicas e privadas das cidades brasileiras de Juazeiro-BA (53\%) e Petrolina-PE $(47 \%)$. Os resultados da análise feita pelo software G Power 3.1. indicam que esse tamanho amostral foi adequado para realização das análises pretendidas $(\mathrm{t} \geq 1,98 ; \pi \geq .98 ; p<.05)$.

\section{Instrumentos}

O instrumento utilizado foi o ICM Jr., versão $\mathrm{B}$, traduzido e adaptado para o português brasileiro, já empregado no primeiro estudo, composto por 15 itens distribuídos nas dimensões de Conhecimento da Cognição e Regulação da Cognição.

\section{Procedimentos}

A seleção das escolas, o recrutamento dos alunos e os procedimentos de aplicação e resposta do questionário foram idênticos aos do primeiro estudo. 


\section{Análises estatísticas}

Para a análise fatorial confirmatória, utilizouse o programa AMOS GRAFICS 21.0, destinado aos cálculos de modelagem de equações estruturais (MEE) e realização de Análise Fatorial Confirmatória (AFC). Com o programa AMOS pretendeu-se testar a adequação do modelo bidimensional, o qual, foi previamente observado na análise exploratória. Considerou-se como entrada a matriz de covariâncias, tendo sido adotado o estimador ML (Maximum Likelihood), buscando comprovar, com maior robustez, a estrutura dimensional da medida psicológica em questão.

Para realização da AFC, buscou-se verificar a adequação do modelo quanto a três propostas fatoriais: a unifatorialidade, bifatorialidade ortogonal e bifatorialidade oblíqua (nesta ultima, comparou-se também, a confirmação para o modelo não ajustado e ajustado). A importância em realizar esse tipo de análise estatística se deve por ela ser mais criteriosa e rigorosa do que a análise fatorial dos Principais Componentes (PC), permitindo verificar diretamente uma estrutura fatorial teórica, a partir dos dados obtidos no estudo exploratório.

Alguns indicadores foram usados para avaliar a qualidade de ajuste dos modelos teóricos testados (Byrne, 2011; Jöreskog \& Sörbom, 2006; Van de Vijver \& Leung, 1997; Hair, Black, Anderson, \& Tatham, 2009), tais como: a relação entre o Qui-quadrado e os graus de liberdade ( $\chi^{2} /$ g.1.), Raiz Quadrada Média Residual Saturada $\left(\mathrm{RMR}_{\mathrm{st}}\right)$, Goodness-of-Fit Index (GFI) e o Adjusted Goodness-of-Fit Index (AGFI), Comparative Fit Index (CFI), Tucker-Lewis Index (TLI), Root-Mean-Square Error of Approximation (RMSEA), Expected Cross-Validation Index (ECVI) e Consistent Akaike Information Criterion (CAIC), Akaike's Information Criteria (AIC), Browne-Cudeck Criterion (BCC) e Bayes Information Criterion (BIC). Além disso, realizaram-se o cálculo de confiabilidade composta $(\mathrm{CC})$ e o da variância média extraída (VME). No primeiro indicador exige-se que o nível do escore seja acima de .70, enquanto que no segundo é desejável a observação de escore acima de .50. Por fim, para avaliar os efeitos das variáveis sexo, idade, série e tipo de escola sobre os indicadores de metacognição foi utilizada uma Análise Multivariada de Variância (MANOVA), associada a uma Análise Univariada de Variância (ANOVA). Aplicou-se ainda o teste de Correlação de Spearman, para avaliar a existência de correlações entre as dimensões da Metacognição e a idade.

\section{Resultados}

Com a organização fatorial da escala observada no primeiro estudo como sendo um modelo oblíquo bifatorial com ajustes no erro, procurou-se, a partir da realização da manipulação de modelos fatoriais comparativos (unifatorial, ortogonal com dois fatores não relacionados e oblíquo sem ajustes) avaliá-los em comparação a um modelo oblíquo ajustado, que contempla os fatores Regulação da Cognição e Conhecimento da Cognição. A análise dos dados do Quadro 3 demonstra que este último modelo apresentou indicadores psicométricos melhores do que os observados nos demais modelos testados.

Além disso, outros indicadores parcimoniosos apontaram para um melhor ajuste do modelo oblíquo ajustado, em relação aos demais testados: $\mathrm{AIC}_{\text {modelo4 }}=159.75, \mathrm{BIC}_{\text {modelo4 }}=278.05 \mathrm{e} \mathrm{BCC}$ modelo4 $=165.35 ; \mathrm{AIC}_{\text {modelo3 }}=181.63, \mathrm{BIC}_{\text {modelo3 }}=$ 286,41 e BCC $_{\text {modelo3 }}=186,59 ;$ AIC $_{\text {modelo2 }}=243.74$, $\mathrm{BIC}_{\text {modelo2 }}=345.14$ e BCC $_{\text {modelo2 }}=248.54$; por fim, $\mathrm{AIC}_{\text {modelo1 }}=193.60, \quad \mathrm{BIC}$ modelo1 $=294.99$ e BCC modelo1 $=198.40 .1$

É preciso destacar que o AIC, BIC e BCC, são indicadores comparativos para verificar a melhor organização empírica dos modelos, de forma que quanto menor o valor, melhor o ajustamento da medida (Marôco, 2010). Essa condição de melhor ajuste é reforçada pelos dados apresentados no Quadro 4, no qual se observa que o modelo oblíquo apresentou os melhores resultados em relação aos outros três testados.

Todas as saturações (Lambdas, $\lambda$ ) estiveram dentro do intervalo esperado $\mid 0$ - $1 \mid$, denotando não existir problemas com a estimação proposta, as quais foram estatisticamente diferentes de zero $(t>1.96, \quad p<.05)$, garantindo a qualidade da validade da estrutura fatorial. Importante observar 
Quadro 3. Indicadores de ajuste global para os modelos hipotetizados

\begin{tabular}{|c|c|c|c|c|c|c|c|c|c|}
\hline \multirow{2}{*}{$\begin{array}{l}\text { Modelos teóricos } \\
\text { testados }\end{array}$} & \multicolumn{9}{|c|}{ Indicadores de ajuste analisados } \\
\hline & $\chi^{2} / g 1$ & $\mathrm{RMR}_{\mathrm{st}}$ & GFI & AGFI & CFI & TLI & $\begin{array}{l}\text { RMSEA } \\
\text { (intervalo) }\end{array}$ & CAIC & $\begin{array}{c}\text { ECVI } \\
\text { (intervalo) }\end{array}$ \\
\hline Unifatorial & 1.48 & .09 & .92 & .89 & .88 & .88 & $\begin{array}{c}.04 \\
(.02-.05)\end{array}$ & 324.99 & $\begin{array}{c}.90 \\
(.77-1.06)\end{array}$ \\
\hline Ortogonal bifatorial & 2.04 & .18 & .90 & .87 & .77 & .74 & $\begin{array}{c}.07 \\
(.06-.08)\end{array}$ & 375.14 & $\begin{array}{c}1.13 \\
(.98-1.33)\end{array}$ \\
\hline $\begin{array}{l}\text { Bifatorial oblíquo } \\
\text { sem ajuste }\end{array}$ & 2.43 & .12 & .89 & .90 & .90 & .91 & $\begin{array}{c}0.06 \\
(.05-.07)\end{array}$ & 327.41 & $\begin{array}{c}.84 \\
(.71-.99)\end{array}$ \\
\hline $\begin{array}{l}\text { Bifatorial oblíquo } \\
\text { ajustado }\end{array}$ & 1.06 & .05 & .95 & .92 & .99 & .99 & $\begin{array}{c}.02 \\
(.00-.02)\end{array}$ & 313.05 & $\begin{array}{c}.74 \\
(.72-.88)\end{array}$ \\
\hline
\end{tabular}

Quadro 4. Indicadores de validade e confiabilidade das dimensões do ICM_Jr

\begin{tabular}{|c|c|c|c|c|c|}
\hline$\xi$ (construto) & $\begin{array}{c}\text { X (variáveis) } \\
\text { [itens] }\end{array}$ & $\lambda$ & $\varepsilon($ erros $)$ & $\mathrm{CC}$ & VME \\
\hline \multirow{9}{*}{ Regulação da cognição } & Item 6 & .59 & .10 & \multirow{9}{*}{.92} & \multirow{9}{*}{.58} \\
\hline & Item 7 & .61 & .38 & & \\
\hline & Item 8 & .52 & .10 & & \\
\hline & Item 9 & .57 & .32 & & \\
\hline & Item 10 & .59 & .35 & & \\
\hline & Item 14 & .54 & .29 & & \\
\hline & Item 15 & .57 & .22 & & \\
\hline & Item 17 & .53 & .20 & & \\
\hline & Item 18 & .53 & .28 & & \\
\hline \multirow{6}{*}{ Conhecimento da cognição } & Item 1 & .47 & .07 & \multirow{6}{*}{.89} & \multirow{6}{*}{.57} \\
\hline & Item 2 & .45 & .12 & & \\
\hline & Item 3 & .56 & .21 & & \\
\hline & Item 5 & .41 & .17 & & \\
\hline & Item 11 & .51 & .26 & & \\
\hline & Item 13 & .55 & .30 & & \\
\hline
\end{tabular}

Nota. $\xi=$ construto psicológico da metacognição; : $\chi=$ variáveis (itens); $\lambda=$ Escores fatoriais da estrutura; $\varepsilon$ (erros) $=$ Erros de medida da estrutura.

ainda a associação Lambdas $(\lambda)$ positivas entre os fatores e seus respectivos itens (variando de $.41 \mathrm{a}$ .61) e que também apresentaram uma relação Phi ( $\Phi)$ positiva, acima de $.30(\Phi=.78)$ entre os fatores do Conhecimento da Cognição e Regulação da Cognição. Esse resultado foi confirmado ao se observar as estimativas de predição, a partir da análise de regressão revelada para o modelo oblíquo, ao identificar as variáveis significativas e a razão critério que estiveram dentro do que é estatisticamente exigido ( $\mathrm{z} \geq 1.96, p<.01)$, tendo sido todos significativos. Bem como, revelaram não haver uma multicolinearidade, pois observou-se que o VIF (definida em português como Inflação da Variância do Fator - IVF), com base no fator de tolerância entre as variáveis (este fator, deverá apresentar escores que não excedam um valor acima de 5) variou de 1,10 a $1,50 \quad(p<.01)$, revelando não existir multicolinearidade, condição que contribui para a estimação qualitativa do modelo pretendido.

Observou-se (Quadro 4) que para as dimensões do ICM Jr., o CC e o VME, estiveram acima do exigido na literatura, isto é, para o Conhecimento da Cognição observou-se um CC de .89, enquanto que para a Regulação da 
Cognição o CC foi de .92. Em relação ao VME, este foi de .57 para o Conhecimento da Cognição e de .58 para a Regulação da Cognição. Tais resultados evidenciam tanto a confiabilidade quanto a validade convergente do construto avaliado, justificando a adequabilidade da estrutura fatorial do ICM-Jr, de forma confiável e com segurança fatorial para amostras com características semelhantes àquelas investigadas nos dois estudos ora apresentados.

Para verificar os efeitos da idade, série, sexo e tipo de escola sobre a Metacognição e seus componentes foi aplicada uma Análise de Variância Multivariada, com a idade sendo categorizada segundo os critérios da OMS (2007): 12 a 14 anos (período inicial da adolescência), 15 a 16 anos (período intermediário da adolescência) e 17 a 18 (período final da adolescência). Além disso, os estudantes foram categorizados em função do nível de escolarização como pertencentes ao nível médio ou fundamental. Os resultados desta análise revelaram que houve efeitos significativos da interação entre idade e nível de escolarização sobre o escore geral de Metacognição $\quad[\mathrm{F}(1, \quad 209)=7.71 ; \quad p=.006$; $\left.\eta_{\mathrm{p}}{ }^{2}=0.038\right]$ e sobre a dimensão regulação da Cognição $\left[\mathrm{F}(1,211)=8.97 ; p=.003 ; \eta_{\mathrm{p}}^{2}=.04\right]$.

Buscando-se aprofundar a compreensão sobre esses resultados foram conduzidas análises univariadas de variância (ANOVA), como followup tests as quais demonstraram que no período intermediário da adolescência (15 a 16 anos) os estudantes do Ensino Médio obtiveram escores mais elevados de Metacognição $[\mathrm{F}(1,82)=9.81$; $\left.p=.02 ; \eta_{\mathrm{p}}{ }^{2}=.10\right]$ e de Regulação da Metacognição do que os estudantes de Ensino Fundamental $[\mathrm{F}(1$, 83)=5.79; $\left.p=.01 ; \quad \eta_{\mathrm{p}}{ }^{2}=.06\right]$. As médias e os desvios-padrões observados foram os seguintes: Ensino Fundamental $\left(\mathrm{M}_{\text {Metacognição }}=50.46 \mathrm{e}\right.$ $\mathrm{dp}=7.04 ; \mathrm{M}_{\text {Regulação da Cognição }}=27.38 \mathrm{e} \mathrm{dp=5.22)} \mathrm{e}$ Ensino Médio $\left(\mathrm{M}_{\text {Metacognição }}=56.46 ; \mathrm{dp}=8.22 \mathrm{e}\right.$ $\mathrm{M}_{\text {Regulação da Cognição }}=\mathrm{M}=30.95 ; \mathrm{dp}=6.47$ ).

Por fim, o teste de Pearson apontou para existência de correlações significativas entre idade e o escore global de Metacognição ( $\mathrm{r}=.33 ; p<.01)$, o Conhecimento da Cognição $(\mathrm{r}=.23 ; p=.03)$ e a Regulação da Metacognição ( $\mathrm{r}=.31 ; p<.01)$, sugerindo que quanto mais velhos os adolescentes, maior o desenvolvimento metacognitivo.

\section{Discussão}

O objetivo principal dessa pesquisa foi traduzir, adaptar e produzir evidências de validade do ICM Jr. (versão B) ao contexto brasileiro e verificar o perfil metacognitivo dos alunos das séries finais do ensino fundamental e dos três anos do ensino médio. A partir dos resultados das análises fatoriais exploratória e confirmatória foram produzidas evidências de validade da versão adaptada para o contexto brasileiro do ICM Jr.

No estudo original de Sperling et al. (2002), a variância total explicada foi de $36 \%$ e a consistência interna da escala foi de .82 , realçando que os fatores encontrados avaliam tanto a Regulação da Cognição como o Conhecimento da Cognição, resultados similares aqueles obtidos nos estudos de Schraw e Denninson (1994), Sperling, Howard, Staley e DuBois (2004). Quanto ao trabalho de Gonçalves et al. (2011), a variância total explicada na versão Portuguesa do ICM-Jr. foi de $42.5 \%$ e o Alfa de .85 .

Tal como observado no trabalho de Gonçalves et al. (2011) em Portugal, a versão adaptada no presente estudo também não reteve todos os itens originalmente propostos por Sperling et al. (2002). Por outro lado, a estrutura fatorial aqui observada se coaduna com aquela obtida nas pesquisas com estudantes Portugueses e Estadunidenses. A este respeito, é importante destacar que, apesar das Análise Fatoriais Exploratórias conduzidas por estes autores terem apontado para possibilidade de aceitação de uma estrutura dimensional que engloba mais do que dois fatores, eles reconhecem que seria mais adquado a adoção de um modelo parcimonioso com dois ou até mesmo um único fator. Essa recomendação vai ao encontro do que foi observado empiricamente por Ribeiro et al. (2016), quando da validação do MAI em Portugal, assim como nos resultados do nosso segundo estudo, quando se procedeu à Análise Fatorial Confirmatória, a qual representou, portanto, um avanço metodológico em relação às pesquisas anteriores.

Assim, previa-se que o modelo oblíquo ajustado seria o mais favorável, pois um dos pressupostos das teorias da metacognição é que os dois componentes Regulação da Cognição e Conhecimento da Cognição estão mutuamente correlacionados (Ribeiro et al., 2016). Essa 
predição foi confirmada na AFC, que produziu evidências para a bifatorialização da escala, em um modelo oblíquo ajustado, assim como Sperling et al. (2002) sugerem.

Destaca-se ainda que a consistência interna produzida a partir da aplicação do instrumento brasileiro foi aceitável e comparável àquela observada nas versões do ICM-Jr. administradas por Sperling et al. (2002) e por Gonçalves et al. (2011). De forma geral, estes dados indicam que o instrumento é adequado para mensurar a Metacognição em adolescentes brasileiros, pois este foi capaz de avaliar suas duas subdimensões, revelando indicadores psicométricos aceitáveis, conforme literatura psicométrica (Hair et al., 2009; Byrne, 2011; Valentini \& Damásio, 2016).

No que se refere aos resultados decorrentes do perfil metacognitivo dos participantes, foi possível observar que quanto mais velhos, maior era o desenvolvimento metacognitivo dos estudantes. Segundo Flavell (1981), Paris e Lindauer (1982), esse resultado é explicado pelo fato das crianças serem bastante limitadas no seu conhecimento e também na consciência dos fenômenos cognitivos, e que elas não têm a percepção dos benefícios da utilização de estratégias metacognitivas na execução de tarefas. Por esta razão é que só tardiamente verificar-se-ia o aparecimento desse atributo do pensamento formal (Garner \& Alexander, 1989).

Os resultados observados nesta pesquisa estão em concordância com os achados de Bryce e Whitebread (2012), que verificaram que na metacognição, os alunos mais velhos possuem um maior monitoramento de controle metacognitivo. Da mesma forma, vão ao encontro do estudo realizado por Oliveira, Boruchovitch e Santos (2009) em alunos do ensino fundamental, com idade entre 7 e 16 anos, no qual se verificou que os alunos mais velhos pontuaram mais em relação aos alunos mais novos.

Em relação aos fatores que influenciam o desenvolvimento da metacognição, Kurtz e Borkowski (1987) e Ribeiro (2003) supõem que em um primeiro momento esta habilidade seja determinada pelo ambiente familiar. À medida que a criança avança nos estudos na escola, se torna mais provável que a atividade metacognitiva seja influenciada pelo estilo de ensino dos professores em conjunto com as experiências escolares. Trata-se de um processo de percepção acerca do próprio aprendizado, pois ao avançar pelas séries escolares, maior será a percepção do aluno para verificar o que não foi compreendido acerca do novo conteúdo. Desta forma, os resultados reforçam a tese de que a escolarização pode contribuir com a metacognição, permitindo que os estudantes desenvolvam mecanismos de autorregulação do seu processo de aprendizagem, tal como proposto por Guterman (2003).

Salienta-se que os dados encontrados foram pontuais e não podem ser generalizáveis, visto que o trabalho foi realizado em grupos heterogêneos, não havendo controle estritamente rigoroso das variáveis tipo de escola, cidades (sistemas educacionais distintos), idades e nível escolar e isso pode ter contribuído para produção de um resultado significativo, mas que talvez não reflita o real papel que essas variáveis têm para Metacognição.

A este respeito é importante questionar que o sistema de ensino nas duas cidades pesquisadas pode ter contribuído sobremaneira para os resultados, pois boa parte dos estudantes mais novos estavam matriculados em uma cidade, enquanto os mais velhos em outra. Dessa forma, em verificação ao sistema IDEB (Índice de Desenvolvimento da Educação Básica) pôde-se observar que as escolas onde se desenvolveu a pesquisa apresentam índices diferentes em relação ao resultado obtido e meta atingida, sendo que apenas uma das escolas pesquisadas conseguiu atingir a meta estabelecida pelo IDEB (INEP, 2016), o que reforça a tese a respeito da possível influência da variável cidade.

Conforme Rosa e Alves Filho (2009) apontam, o sistema de ensino tem o papel fundamental de desenvolver mecanismos que favorecem a aprendizagem, do aprender a aprender e de fazê-lo com autonomia. Neste sentido, estudos futuros devem buscar avaliar melhor a relação entre o sistema educacional do qual o estudante participa e o seu desenvolvimento metacognitivo.

Dessa forma, recomenda-se que, em estudos futuros, seja melhor explorada a relação entre a motivação e a metacognição no ensino fundamental e médio, percebendo-se a necessidade de controlar melhor as variáveis e a similaridade dos estratos (em termos educacionais 
e sociodemográficos) a serem comparados durante o processo de amostragem, para que se possa isolar os efeitos da idade, sexo e escolarização, visto que existem poucas investigações nessa área no âmbito nacional.

\section{Considerações Finais}

Os dados encontrados nesse estudo apontam para a adequação da versão adaptada do ICM-Jr. para avaliação da metacognição em adolescentes do Brasil. Esse fato é de extrema importância, pois esse instrumento pode contribuir com outras áreas do conhecimento, como a educação, permitindo investigar melhor a relação entre metacognição, aprendizagem e outras variáveis sociocognitivas, bem como contribuir com elaboração de programas de intervenção voltados ao desenvolvimento da metacognição nesta faixa etária. Além disso, a dimensionalidade da escala corrobora o modelo bifatorial de Sperling et al. (2002).

Podem-se destacar algumas limitações importantes deste estudo, as quais podem ser vistas como oportunidades para pesquisas futuras. Em termos metodológicos, cabe destacar que, no presente estudo, a metacognição foi mensurada pelo ICM Jr., utilizado pela primeira vez no Brasil. A amostra da pesquisa foi restrita às cidades de Juazeiro-BA e Petrolina-PE, mesmo estas cidades pertencendo a estados diferentes, geograficamente elas se encontram muito próximas. Assim, estudos futuros poderiam aplicar o instrumento validado em outros contextos regionais, dando maior representatividade à população brasileira, para analisar os domínios atitudinais do construto e corroborar ou refutar os achados deste estudo. Além disso, novas pesquisas podem buscar avaliar as relações entre a metacognição e outros componentes cognitivos importantes para o aprendizado e resolução de problemas, tais como as funções executivas.

\section{Referências}

Akin, A., Abaci, R., \& Çetin, B. (2007). The validity and reliability of the Turkish version of the metacognitive awareness inventory. Educational Sciences: Theory \& Practice,
7(2), 671-678. Retrieved from: http://oldsite.estp.com.tr/pdf/en/9acec09565cc a0166f8d1b4f7a17a1b7kinen.pdf

Ayersman, D. J. (1995). Effects of knowledge representation format and hypermedia instruction on metacognitive accuracy. Computers in human behavior, 11, 533-555. doi:10.1016/0747-5632(95)80016-2

Boruchovitch, E. (1999). Estratégias de aprendizagem e desempenho escolar: Considerações para a prática educacional. Psicologia: Reflexão e Crítica, 12, 361-376. doi:10.1590/S0102-79721999000200008.

Boruchovitch, E., Santos, A. A. A. D., Costa, E. R. D., Neves, E. R. C., Cruvinel, M., Primi, R., \& Guimarães, S. E. R. (2006). A construção de uma escala de estratégias de aprendizagem para alunos do ensino fundamental. Psicologia: Teoria e Pesquisa, 22, 297-304. doi:10.1590/S0102-37722006000300006

Bryce, D., \& Whitebread, D. (2012). The development of metacognitive skills: Evidence from observational analysis of young children's behavior during problemsolving. Metacognition Learning, 7, 197-217. doi:10.1007/s11409-012-9091-2

Byrne, B. M. (2011). Structural Equation Modeling with Mplus: Basic Concepts, Applications, and Programming (Multivariate Applications Series). New York: Routledge.

Bustos, A. P. H., Bravo, G. J. V., \& León, M. G. (2014). Validación del instrumento 'inventario de habilidades metacognitivas (MAI)'con estudiantes colombianos. Praxis \& Saber, 5, 56-74. doi:10.19053/22160159.3022

Cannon-Bowers, J. A., Rhodenizer, L., Salas, E., \& Bowers, C. A. (1998). A framework for understanding pre-practice conditions and their impact on learning. Personnel Psychology, 51(2), 291-320. doi:10.1111/j.1744-6570.1998.tb00727.x

Davidson, E., Deuser I, \& Sternberg, R. (1994). The role of metacognition in problem solving. In: Metcalfe, J., \& Shimamura, A. (Eds.). Metacognition: Knowing about knowing (pp. 207-226). Cambridge, MA: The MIT Press.

Dunlosky, J., \& Metcalfe, J. (2009). Metacognition. Thousand Oaks, CA: Sage Publications. 
Fabriga, L. R., Wegener, D. T., MacCallum, R. C., \& Strahan, E.J. (1999). Evaluating the use of exploratory factor analysis in psychological research. Psychological Methods, 4, 272-299. doi:10.1037/1082-989x.4.3.272

Faul, F., Erdfelder E., Buchner A., \& Lang A.-G. (2009). Statistical power analyses using $\mathrm{G}^{*}$ Power 3.1: tests for correlation and regression analyses. Behavior Research Methods, $\quad 41, \quad 1149-1160$. doi:10.3758/brm.41.4.1149

Figueira, A. P. C (2003). Metacognição e seus contornos. Revista Iberoamericana de Educacion, 33 (1) 1-20. Retrieved from: http://www.rieoei.org/deloslectores/446Couce iro.pdf

Flavell, J. H., \& Wellman, H. M. (1977). Metamemory. In: Kail, R. V., \& Hagen, J. H. (Eds.), Perspectives on the development of memory and cognition (pp. 3-33). Hillsdale: Lawrence Erlbaum Associates.

Flavell, J. H. (1979). Metacognition and cognitive monitoring: A new area of cognitivedevelopmental inquiry. American Psychologist, 34, 906-911. doi:10.1037/0003066X.34.10.906

Flavell, J. H. (1981). Monitoring social cognitive enterprises: something else that may develop in the area of social cognition. In: Social cognitive development: Frontiers and possible futures (pp. 272-287). Flavell, J. H., \& Ross, L. (Eds.). Cambridge, MA: Cambridge University Press.

Garner, R., \& Alexander, P. A. (1989). Metacognition: Answered and unanswered questions. Educational psychologist, 24, 143158. doi:10.1207/s15326985ep2402_2

Gonçalves, J., Fidalgo, Z., \& Martins, M.A. (2011). Avaliação do desenvolvimento metacognitivo de estudantes entre o sexto e nono ano de escolaridade. Paper presented at the XI Congresso Internacional GalegoPortugués de Psicopedagoxía. A Coruña. Retrieved from: http://uipcde.ispa.pt/ficheiros/areas_utilizador/ user16/goncalves_j._fidalgo_z._alves_martins _m._2011_avaliacao_do_desenvolvimento_m etacognitivo_.pdf

Guterman, E. (2003). Integrating written metacognitve awarencess guidance as a psychological tool to improve student performance. Learning and Instruction, 13, 633-651. doi:10.1016/s0959-4752(02)00070-1

Hair, J. F., Black, W. C., Anderson, R. E., \& Tatham, R. L. (2009). Análise multivariada de dados. Porto Alegre. Bookman Editora.

Hayton, J. C., Allen, D. G., \& Scarpello, V. (2004). Factor retention decisions in exploratory factor analysis: A tutorial on parallel analysis. Organizational Research Methods, 7, 191-205. doi:10.1177/1094428104263675

Instituto Nacional de Estudos e Pesquisas Educacionais Anisio Teixeira (2016). Indice de Desenvolvimento da Educação Básica. Retrieved from: http://ideb.inep.gov.br/resultado/resultado/res ultado.seam?cid=727962

Joly, M. C. R. A. (2006). Escala de estratégias de leitura para etapa inicial do ensino fundamental. Estud. psicol. (Campinas), 23, 271-278. doi:10.1590/S0103-166X2006000300006.

Joly, M. C. R. A., Santos, L. M., \& Marini, J. A. S. (2006). Uso de estratégias metacognitivas de leitura por alunos do ensino médio. Paidéia, 16, 205-212. doi:10.1590/s0103-863x2006000200008

Jöreskog, K. G., \& Sörbom, D. (2006). LISREL 8.80 for Windows [Computer Software]. Lincolnwood, IL: Scientific Software International, Inc.

Kline, R. B. (2016). Principles and Practice of Structural Equation Modeling, $4^{\text {th }}$ Edit. New York, NY. Guilford Press.

Kurtz, B. E., \& Borkowski, J. G. (1987). Development of strategic skills in impulsive and reflective children: A longitudinal study of metacognition. Journal of Experimental Child Psychology, 43, 129-148. doi:10.1016/0022-0965(87)90055-5

Kuhn, D. (2000). Metacognitive development. Current directions in psychological science, 9 (5), 178-181. doi:10.1111/1467-8721.00088

Ledesma, R. D., \& Valero-Mora, P. (2007). Determining the number of factors to retain in EFA: an easy-to-use computer program for carrying out parallel analysis. Practical assessment, research \& evaluation, 12 (2), 111. Retrieved from: https://pareonline.net/pdf/v12n2.pdf 
Lima Filho, R. N., \& Bruni, A. L. (2015). Metacognitive Awareness Inventory: Translation and Validation from a Confirmatory Analysis. Psicologia: Ciência e Profissão, 35, 1275-1293. doi:10.1590/19823703002292013

Manning, B. H., Glasner, S. E., \& Smith, E. R. (1996). The self-regulated learning aspect of metacognition: A component of gifted education. Roeper Review, 18, 217-223. doi:10.1080/02783199609553741

Marini, J. A. D. S., \& Joly, M. C. R. A. (2008). A leitura no Ensino Médio e o uso das estratégias metacognitivas. Estudos $e$ Pesquisas em Psicologia, 8 (2), 505-522. Retrieved from: http://www.revispsi.uerj.br/v8n2/artigos/pdf/v 8n2a24.pdf

Marôco, J. (2010). Análise de equações estruturais. Lisboa, Portugal: Report Number.

Narang, D., \& Saini, S. (2013). Metacognition and academic performance of rural adolescents. Studies on Home and Community Science, 7, 167-175. doi:10.1080/09737189.2013.11885409

O'Connor, B. P. (2000). SPSS and SAS programs for determining the number of components using parallel analysis and Velicer's MAP test. Behavior Research Methods, 32, 396402. doi:10.3758/bf03200807

Oliveira, K. L. de, Boruchovitch, E., \& Santos, A. A. A. (2009). Estratégias de aprendizagem e desempenho acadêmico no ensino fundamental: Evidências de validade. Psicologia: Teoria e Pesquisa, 25, 531-536. doi:10.1590/s0102-37722009000400008

Organização Mundial de Saúde - OMS (2007). Growth reference data for 5-19 years. Retrieved from: http://www.who.int/growthref/who2007_bmi_ for_ag e/en/index.html

Paris, S. G., \& Lindauer, B. K. (1982). The development of cognitive skills during childhood. In: Wolman, B. (Ed.). Handbook of developmental psychology (pp. 333-349). Englewood- Cliffs, NJ; Prentice-Hall.

Pasquali, L. (2003). Psicometria: teoria dos testes na psicologia e na educação. São Paulo, SP. Vozes.

Pascualon, J. F. (2011). Escala de avaliação da metacognição infantil: Elaboração de itens e análise dos parâmetros psicométricos (Dissertação de Mestrado). Universidade Federal de São Carlos, São Carlos, SP.

Ribeiro, C. (2003). Metacognição: um apoio ao processo de aprendizagem. Psicologia: reflexão e crítica, 16, 109-116. doi:10.1590/s0102-79722003000100011

Ribeiro, R. B., Dias, O., Oliveira, I. M., Miranda, P., Ferreira, G., Saraiva, M., Paulo, R., \& Cadime, I. (2015). Adaptação e validação da escala reading strategy use para a população portuguesa. Revista Iberoamericana de Diagnóstico y Evaluación - e Avaliação Psicológica, 40, 25-36. Retrieved from: http://www.redalyc.org/pdf/4596/4596454320 04.pdf

Ribeiro, R. B., Simões, M. R., \& Almeida, L. D. S. (2016). Metacognitive awareness inventory (MAI): Adaptação e validação da versão portuguesa. Revista Iberoamericana de Diagnóstico y Evaluación - e Avaliação Psicológica, $\quad 42, \quad 143-159$. doi:10.21865/ridep42_145

Rosa, C. W., \& Alves Filho, J. P. (2009). A dimensão metacognitiva na aprendizagem em física: Relato das pesquisas brasileiras. Revista Electrónica de Enseñanza de las Ciencias, 8 (3), 1117-1139. Retrieved from: https://reec.educacioneditora.net/volumenes/v olumen8/ART19_Vol8_N3.pdf

Schraw, G. (1998). Promoting general metacognitive awareness. Instructional Science, 26, 113-125. doi:10.1023/A:1003044231033

Schraw, G., \& Dennison, R. S. (1994). Assessing metacognitive awareness. Contemporary Educational Psychology,19, 460-75. doi:10.1006/ceps.1994.1033

Sperling, R. A., Howard, B. C., Miller, L. A., \& Murphy, C. (2002). Measures of children's knowledge and regulation of cognition. Contemporary educational psychology, 27, 51-79. doi:10.1006/ceps.2001.1091

Sperling, R. A., Howard, B. C., Staley, R., \& Dubois, N. (2004). Metacognition and selfregulated learning constructs. Educational Researchand and Evaluation, 10 (2), 117-139. Retrieved from: http://www4.ncsu.edu/ jlnietfe/Metacog_Arti cles_files/Sperling,\%20Howard,\%20Staley,\% 20\%26\%20DuBois\%20\%282004\%29.pdf 
Swanson, H. L. (1990). Influence of metacognitive knowledge and aptitude on problem solving. Journal of educational Psychology, 82, 306. doi:10.1037/0022-0663.82.2.306

Tabachnick, B. G., \& Fidell, L. S. (2012). Using multivariate statistics. Needham Boston, MA: Pearson.

Valentini, F., \& Damásio, B. F. (2016). Variância média extraída e confiabilidade composta: indicadores de precisão. Psicologia: Teoria e Pesquisa, 32, 1-7. doi:10.1590/0102-3772e322225

Van De Vijver, F., \& Leung, K. (1997). Methods and data analysis for cross-cultural research. Thousand Oaks, CA: Sage Publications.

Zimmerman, B. J., \& Pons, M. M. (1986). Development of a structured interview for assessing student use of self-regulated learning strategies. American Educational Journal, 23, 614-628. doi:10.3102/00028312023004614 